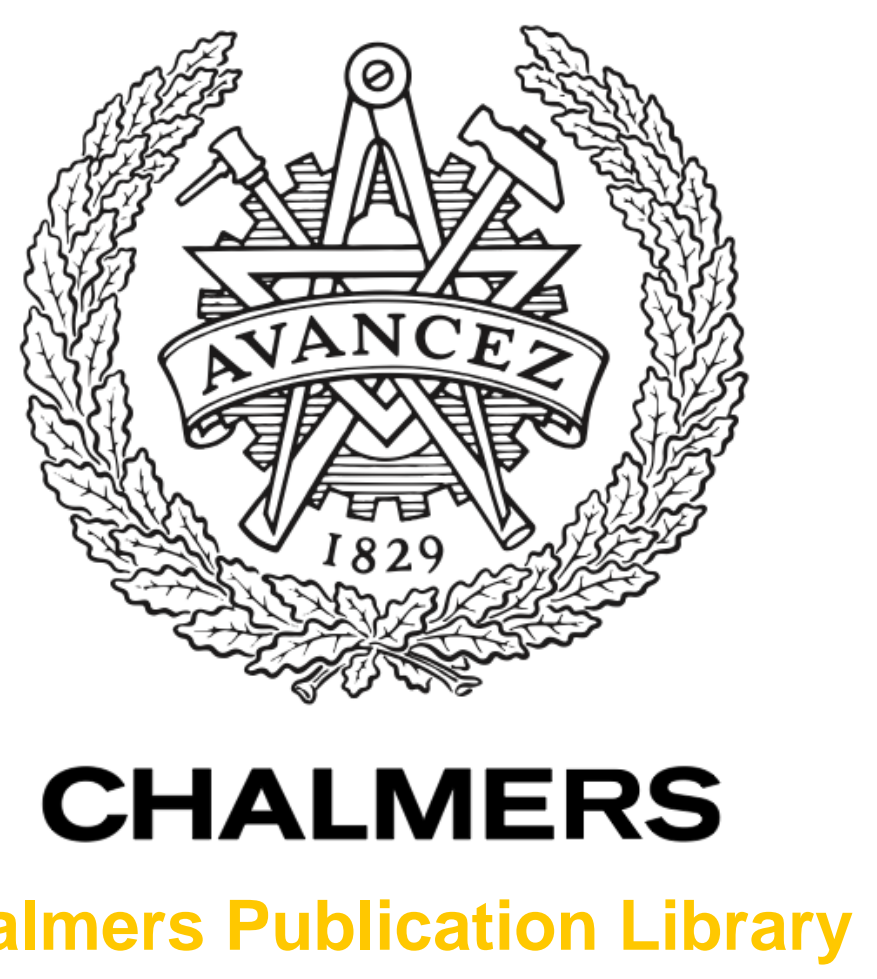

Chalmers Publication Library

\title{
CARBON MONOXIDE IN THE COLD DEBRIS OF SUPERNOVA 1987A
}

This document has been downloaded from Chalmers Publication Library (CPL). It is the author's version of a work that was accepted for publication in:

Astrophysical Journal Letters (ISSN: 2041-8205)

Citation for the published paper:

Kamenetzky, J. ; McCray, R. ; Indebetouw, R. et al. (2013) "CARBON MONOXIDE IN

THE COLD DEBRIS OF SUPERNOVA 1987A". Astrophysical Journal Letters, vol. 773(2),

http://dx.doi.org/10.1088/2041-8205/773/2/134

Downloaded from: http://publications.lib.chalmers.se/publication/185863

Notice: Changes introduced as a result of publishing processes such as copy-editing and formatting may not be reflected in this document. For a definitive version of this work, please refer to the published source. Please note that access to the published version might require a subscription.

Chalmers Publication Library (CPL) offers the possibility of retrieving research publications produced at Chalmers University of Technology. It covers all types of publications: articles, dissertations, licentiate theses, masters theses, conference papers, reports etc. Since 2006 it is the official tool for Chalmers official publication statistics. To ensure that Chalmers research results are disseminated as widely as possible, an Open Access Policy has been adopted.

The CPL service is administrated and maintained by Chalmers Library. 


\title{
CARBON MONOXIDE IN THE COLD DEBRIS OF SUPERNOVA 1987A
}

\author{
J. Kamenetzky ${ }^{1}$, R. McCray ${ }^{1}$, R. Indebetouw ${ }^{2,3}$, M. J. Barlow ${ }^{4}$, M. Matsuura ${ }^{4}$, M. Baes ${ }^{5}$, J. A. D. L. Blommaert ${ }^{6,7}$,

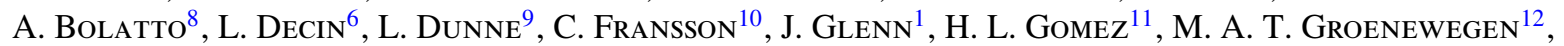

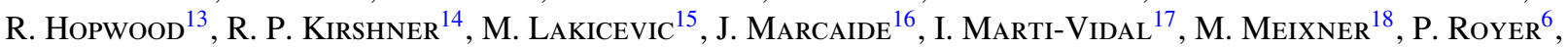 \\ A. SoderberG ${ }^{14}$, G. Sonneborn ${ }^{19}$, L. Staveley-Smith ${ }^{20,21}$, B. M. Swinyard ${ }^{4}$, G. Van de Steene ${ }^{12}$, P. A. M. van Hoof ${ }^{12}$, \\ J. Th. VAN LoON ${ }^{15}$, J. YATES ${ }^{4}$, AND G. ZANARDO ${ }^{20}$ \\ ${ }^{1}$ Department of Astrophysical and Planetary Sciences, University of Colorado at Boulder, UCB 391, Boulder, CO 80309, USA \\ ${ }^{2}$ Department of Astronomy, University of Virginia, P.O. Box 400325, Charlottesville, VA 22903, USA \\ ${ }^{3}$ National Radio Astronomy Observatory, 520 Edgemont Road, Charlottesville, VA 22903, USA \\ ${ }^{4}$ Department of Physics and Astronomy, University College London, Gower Street, London WC1E 6BT, UK \\ ${ }^{5}$ Sterrenkundig Observatorium, Universiteit Gent, Krijgslaan 281 S9, B-9000 Gent, Belgium \\ ${ }^{6}$ Instituut voor Sterrenkunde, KU Leuven, Celestijnenlaan 200D BUS 2401, B-2001 Leuven, Belgium \\ ${ }^{7}$ Department of Physics and Astrophysics, Vrije Universiteit Brussel, Pleinlaan 2, B-1050 Brussels, Belgium \\ ${ }^{8}$ Department of Astronomy, University of Maryland, College Park, MD 20742, USA \\ ${ }^{9}$ Department of Physics and Astronomy, University of Canterbury, Private Bag 4800, Christchurch 8410, NZ \\ ${ }^{10}$ Department of Astronomy, The Oskar Klein Centre, Stockholm University, AlbaNova, SE-106 91 Stockholm, Sweden \\ ${ }^{11}$ School of Physics and Astronomy, Cardiff University, The Parade, Cardiff CF24 3AA, UK \\ ${ }_{12}$ Royal Observatory of Belgium, Ringlaan 3, B-1180 Brussels, Belgium \\ ${ }^{13}$ Physics Department, Imperial College London, London SW7 2AZ, UK \\ ${ }^{14}$ Harvard College Observatory, 60 Garden Street, Cambridge, MA 02138, USA \\ ${ }^{15}$ Lennard-Jones Laboratories, Keele University, Staffordshire ST5 5BG, UK \\ ${ }^{16}$ Universidad de Valencia, C/Dr. Moliner 50, E-46100 Burjassot, Spain \\ 17 Onsala Space Observatory, SE-439 92 Onsala, Sweden \\ ${ }^{18}$ Space Telescope Science Institute, 3700 San Martin Drive, Baltimore, MD 21218, USA \\ ${ }^{19}$ NASA Goddard Space Flight Center, 8800 Greenbelt Road, Greenbelt, MD 20771, USA \\ ${ }^{20}$ International Centre for Radio Astronomy Research (ICRAR), University of Western Australia, Crawley, WA 6009, Australia \\ ${ }^{21}$ ARC CEntre of Excellence for All-sky Astrophysics (CAASTRO), University of Western Australia, Crawley, WA 6009, Australia \\ Received 2013 June 6; accepted 2013 July 22; published 2013 August 7
}

\begin{abstract}
We report spectroscopic and imaging observations of rotational transitions of cold $\mathrm{CO}$ and $\mathrm{SiO}$ in the ejecta of SN1987A, the first such emission detected in a supernova remnant. In addition to line luminosities for the CO $J=$ $1-0,2-1,6-5$, and 7-6 transitions, we present upper limits for all other transitions up to $J=13-12$, collectively measured from the Atacama Large Millimeter Array, the Atacama Pathfinder EXperiment, and the Herschel Spectral and Photometric Imaging REceiver. Simple models show the lines are emitted from at least $0.01 M_{\odot}$ of $\mathrm{CO}$ at a temperature $>14 \mathrm{~K}$, confined within at most $35 \%$ of a spherical volume expanding at $\sim 2000 \mathrm{~km} \mathrm{~s}^{-1}$. Moreover, we locate the emission within $1^{\prime \prime}$ of the central debris. These observations, along with a partial observation of $\mathrm{SiO}$, confirm the presence of cold molecular gas within supernova remnants and provide insight into the physical conditions and chemical processes in the ejecta. Furthermore, we demonstrate the powerful new window into supernova ejecta offered by submillimeter observations.
\end{abstract}

Key words: ISM: supernova remnants - supernovae: individual (SN1987A)

Online-only material: color figures

\section{INTRODUCTION}

The ejecta of supernovae (SNe) encode important information about the SN explosion dynamics, the nucleosynthetic yield, and the formation of interstellar dust and molecules. At optical and X-ray wavelengths, the dominant feature of SN1987A is a rapidly brightening $1^{\prime \prime} 66 \pm 0$ 0'03 (Panagia et al. 1991) ring of "hot spots" where the blast wave is colliding with the innermost of three circumstellar rings (McCray 2007). In the radio, this ring can be seen as two bright lobes of emission ( $\mathrm{Ng}$ et al. 2008; Zanardo et al. 2013). Inside this ring, optical and near-infrared spectra showed that the debris from the progenitor's stellar interior is clumped into chemically distinct fragments, originating from the nucleosynthesis that took place before and during the SN explosion. As the remnant cooled, dust formed and began to obscure the inner ejecta from view at optical wavelengths within the first few years. It was recently estimated that the ejecta of SN1987A contains
0.4-0.7 $M_{\odot}$ of dust at a temperature of $20 \mathrm{~K}$ (Matsuura et al. 2011). Fortunately, observations at far-infrared (FIR) to millimeter wavelengths pierce through the dust, providing a window into the cool interior (Biermann et al. 1992; Lakićević et al. 2011).

A small mass $\left(10^{-3} M_{\odot}\right)$ of hot, vibrationally excited $\mathrm{CO}$ was seen 192 days after the explosion (Liu et al. 1992), but the emission faded below detection limits after $\sim 600$ days, when the gas became too cool to excite vibrational transitions (Liu \& Dalgarno 1995; $700 \mathrm{~K}$ at 800 days). Theoretical models predict that $\mathrm{CO}$ formation would continue after that time, yielding $\sim 0.1 M_{\odot}$ of CO (Cherchneff \& Dwek 2009; Cherchneff \& Sarangi 2011) by 1000 days. Such a mass of CO would have important implications for the evolution of the ejecta, as discussed in Cherchneff \& Sarangi (2011): first, dust synthesis can be limited by the depletion of elements by molecules, and second, $\mathrm{CO}$ is a powerful coolant, and a cooler environment will favor more efficient dust and molecule formation. 


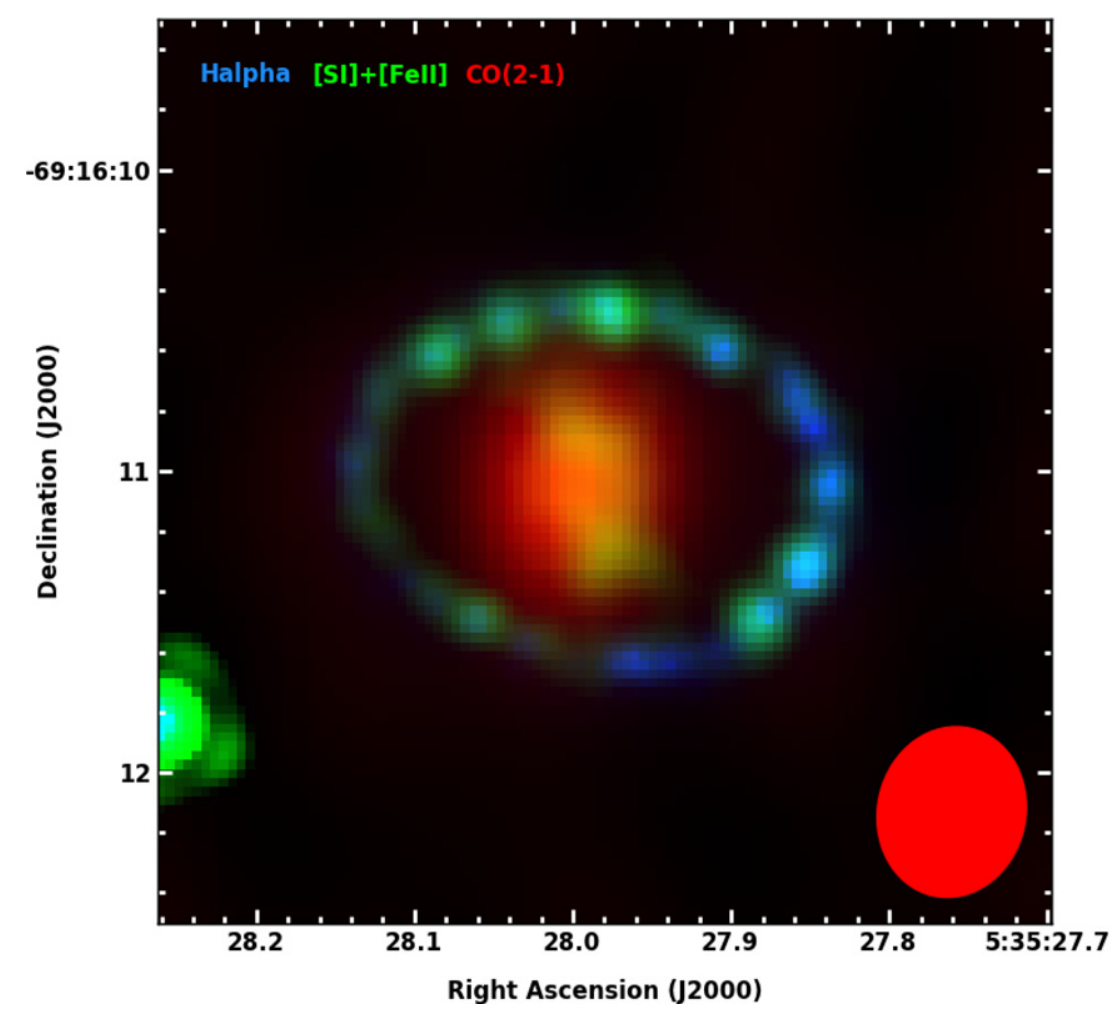

Figure 1. A color composite image of SN1987A. The unresolved ${ }^{12} \mathrm{CO} 2-1$ line emission detected by ALMA is shown in red, and the red ellipse in the corner is the synthesized beam. Also shown are the $\mathrm{H} \alpha$ emission (blue) and [Si I]+[Fe II] $1.644 \mu$ m emission (green in the ring; yellow in the ejecta) observed with the Hubble Space Telescope (Larsson et al. 2013).

(A color version of this figure is available in the online journal.)

The ejecta of at least eight other SNe have been observed in the vibrational CO fundamental $(4.65 \mu \mathrm{m})$ or first overtone $(2.3 \mu \mathrm{m})$ bands, all within the first few years after their explosion (Cherchneff \& Sarangi 2011; see references listed within their Table 1). A notable addition is the $300 \mathrm{yr}$ old Cassiopeia A remnant, where Rho et al. (2012) detected vibrationally excited ${ }^{12} \mathrm{CO}$ with a mass of $\sim 10^{-6} M_{\odot}$ and temperature as high as $900-1400 \mathrm{~K}$. Until now, no direct evidence (via rotational molecular emission) had been found for cold ( $<900 \mathrm{~K})$ molecular gas within SN remnants, and no measurement made between $\sim 2$ and 300 yr post-explosion. With the Atacama Large Millimeter Array (ALMA), we have now imaged the ejecta of SN1987A and, for the first time, detected rotational transitions of $\mathrm{CO}$ inside a $\mathrm{SN}$ remnant.

\section{OBSERVATIONS}

\subsection{ALMA}

SN1987A was observed with ALMA at wavelengths of $2.6 \mathrm{~mm}$ (Band 3) and $1.3 \mathrm{~mm}$ (Band 6) between 2012 April and August. During the 2012 Early Science period, ALMA had only a fraction of its final spatial resolution and sensitivity, but could already obtain synthesized beam spatial resolutions of $\sim 1^{\prime \prime} .6 \times 1^{\prime \prime} .5$ and $0^{\prime} .6 \times 0$ 0.5, respectively. Observation dates and parameters are listed in Table 1. Both bands were observed using Frequency Division Mode, i.e., $488 \mathrm{kHz}$ channels, to enable detection of any narrow emission lines (none were detected), and later averaged to $50 \mathrm{~km} \mathrm{~s}^{-1}$ spectral resolution (corresponding to $16019 \mathrm{MHz}$ channels in Band 3). The bandwidth per spectral window was $1.875 \mathrm{GHz}$. All observations used quasars J0538-440 and J0637-752 as bandpass and phase calibrators, respectively. Callisto was observed as an absolute flux calibrator; the absolute flux scale was then applied to the X352 and $\mathrm{X} 1 \mathrm{c}$ blocks by equating the flux densities of the two quasars to the flux densities calibrated against a solar system object. System temperature and precipitable water vapor (PWV) calibrations were applied before standard bandpass and complex gain (phase) calibration. In the synthesis imaging, the robust parameter was set to 0 , midway between natural and uniform weighting (Briggs 1995).

We have detected emission from the rotational lines of ${ }^{12} \mathrm{CO}$ $J=1-0(115.3 \mathrm{GHz}, 2.6 \mathrm{~mm})$ and $J=2-1(230.6 \mathrm{GHz}$, $1.3 \mathrm{~mm}$ ), as well as the red wing of $\mathrm{SiO} J=5-4$ at 217.1 GHz. Figure 1 is a color composite image showing the ${ }^{12} \mathrm{CO} 2-1$ emission detected by ALMA (red) and other emission $(\mathrm{H} \alpha$ and $[\mathrm{Si} \mathrm{I}]+[\mathrm{Fe} \mathrm{II}])$ for comparison. The origin of the ${ }^{12} \mathrm{CO} J=2-1$ emission, marginally larger in extent than the beam (unresolved), is shown here to be less than $1^{\prime \prime}$ in extent and located at the center of the debris. The spectrum shown in Figure 2 of the center of the remnant includes some continuum emission. However, consistent with observations by Lakićević et al. (2012), this can be identified with the synchrotron emission from the ring. The line profiles are broad (FWHM $\sim 2200 \mathrm{~km} \mathrm{~s}^{-1}$; Table 2), consistent with the nearinfrared [Si I]+[Fe I] lines (Kjær et al. 2010) and ${ }^{12} \mathrm{CO}$ rotationalvibrational models (Liu et al. 1992), confirming an origin from the expanding ejecta (and a constant expansion velocity over time). The lines are narrower than those (4500 to $11,000 \mathrm{~km} \mathrm{~s}^{-1}$ ) of ejecta metal lines observed in the visible (Fransson et al. 


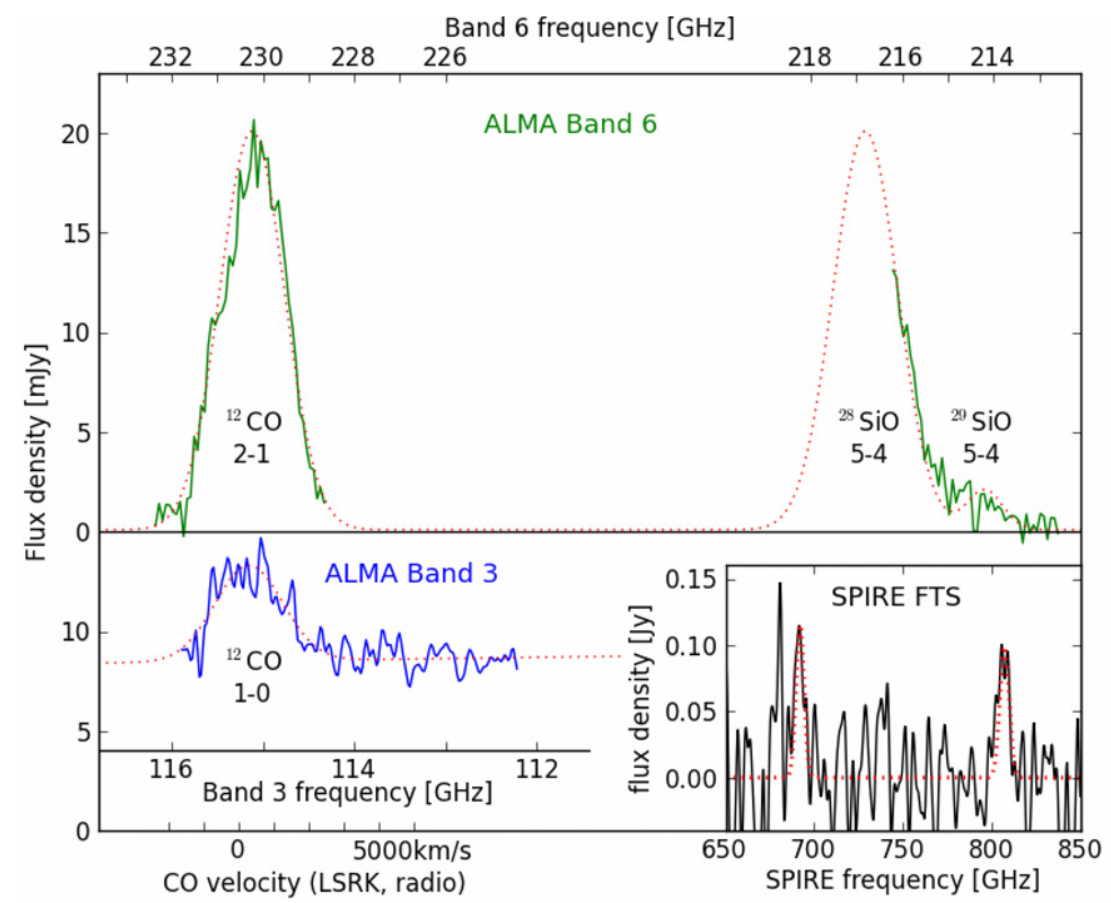

Figure 2. ALMA spectra at the center of SN1987A. The $2.6 \mathrm{~mm}$ Band 3 (bottom left) and the $1.3 \mathrm{~mm}$ Band 6 (top) are plotted with a common velocity axis calculated for the ${ }^{12} \mathrm{CO} 1-0$ and 2-1 lines, respectively. The dotted lines are the best-fit Gaussians to the ${ }^{12} \mathrm{CO}$ lines, ${ }^{28} \mathrm{SiO}$ and ${ }^{29} \mathrm{SiO}$ and a continuum with synchrotron spectral index of -0.8 . A zoomed-in portion of the continuum-subtracted SPIRE spectrum is shown in the bottom right, with the best-fit Gaussians of the $J=6-5$ and $J=7-6$ lines as dotted lines.

(A color version of this figure is available in the online journal.)

Table 1

ALMA Observational Parameters

\begin{tabular}{lcccccccc}
\hline \hline UID (uid://) & Band & $\begin{array}{c}\text { Date } \\
(2012)\end{array}$ & No. of Ant ${ }^{\mathrm{a}}$ & $\begin{array}{c}\text { PWV } \\
(\mathrm{mm})\end{array}$ & $\begin{array}{c}\mathrm{Int}^{\mathrm{b}} \\
(\mathrm{min})\end{array}$ & $\begin{array}{c}\mathrm{RMS}^{\mathrm{c}} \\
\left(\mathrm{mJy} \mathrm{bm}^{-1}\right)\end{array}$ & $\begin{array}{c}\mathrm{BL}_{\mathrm{RMS}}{ }^{\mathrm{d}} \\
(\mathrm{m})\end{array}$ & $\begin{array}{c}\mathrm{BL}_{\max }{ }^{\mathrm{d}} \\
(\mathrm{m})\end{array}$ \\
\hline A002/X3c5ee0/X24b & $3^{\mathrm{e}}$ & Apr 5 & $16 / 18$ & 3.1 & 25 & 0.13 & 220 & 403 \\
A002/X3c7a84/X1c & 3 & Apr 6 & $14 / 17$ & 3.7 & 25 & 0.12 & 225 & 402 \\
A002/X3c8f66/X352 & $6^{\mathrm{f}}$ & Apr 7 & $14 / 17$ & 3.7 & 25 & 0.20 & 225 & 402 \\
A002/X45f1dd/Xd13 & 6 & Jul 15 & $16 / 19$ & 1.3 & 12 & 0.14 & 213 & 402 \\
A002/X494155/X8be & 6 & Aug 10 & $22 / 23$ & 1.4 & 25 & 0.09 & 194 & 402 \\
\hline
\end{tabular}

Notes.

${ }^{a}$ Effective number of antennae after flagging/the total number in the array at the time.

$\mathrm{b}$ Time on the science target, not including calibration overhead.

${ }^{\mathrm{c}}$ RMS (root mean square) noise is measured over a width of $100 \mathrm{~km} \mathrm{~s}^{-1}$.

d Baseline.

e $100.1-103.9$ and $112.2-118.8 \mathrm{GHz}$.

f $212.5-216.3$ and $228.7-232.4 \mathrm{GHz}$.

Table 2

Fitted Line Parameters

\begin{tabular}{lccccc}
\hline \hline Line & $\begin{array}{c}\text { FWHM } \\
\left(\mathrm{km} \mathrm{s}^{-1}\right)\end{array}$ & $\begin{array}{c}\text { Central Velocity } \\
\left(\mathrm{km} \mathrm{s}^{-1}\right)\end{array}$ & $\begin{array}{c}\text { Peak Intensity } \\
\left(\mathrm{mJy} \mathrm{beam}^{-1}\right)\end{array}$ & $\begin{array}{c}\text { Integrated Flux } \\
\left(\mathrm{Jy} \mathrm{km} \mathrm{s}^{-1}\right)\end{array}$ & $\begin{array}{c}\text { Luminosity }^{\mathrm{a}} \\
\left(10^{31} \mathrm{erg} \mathrm{s}^{-1}\right)\end{array}$ \\
\hline${ }^{12} \mathrm{CO} \mathrm{1-0}$ & $2270 \pm 190$ & $300 \pm 100$ & $4.8 \pm 0.3$ & $11.6 \pm 1.2$ & $1.33 \pm 0.14$ \\
${ }^{12} \mathrm{CO} \mathrm{2-1}$ & $2150 \pm 50$ & $390 \pm 20$ & $20.0 \pm 0.7$ & $46.0 \pm 1.9$ & $10.5 \pm 0.4$ \\
\hline
\end{tabular}

Notes. Uncertainties are one standard deviation. In subsequent analysis we added in quadrature a $10 \%$ continuum subtraction error and a $15 \%$ (10\%) absolute flux calibration error for ${ }^{12} \mathrm{CO} 1-0(2-1)$, leading to total line luminosity uncertainties $0.28 \times 10^{31}$ and $1.5 \times 10^{31} \mathrm{erg} \mathrm{s}^{-1}$, respectively.

${ }^{a}$ Luminosities are calculated using a distance of $50 \mathrm{kpc}$ throughout this work (Panagia 1999).

2013), indicating that the ${ }^{12} \mathrm{CO}$ emission originates from a more centrally condensed source.

Although our intention with Band 6 was to observe the ${ }^{12} \mathrm{CO}$ $J=2-1$ line, the red wing of the ${ }^{28} \mathrm{SiO} J=5-4$ line at
217.11 GHz appeared in the upper sideband. If it has the same profile as ${ }^{12} \mathrm{CO} J=2-1$, then the amplitude would be $20 \pm 5$ mJy. Because we do not measure the full flux in the line, we do not conduct further analysis at this time. We see a faint hint 
of the isotopic ${ }^{29} \mathrm{SiO}$ line at $214.39 \mathrm{GHz}$ (at about one-tenth the estimated amplitude of ${ }^{28} \mathrm{SiO}$, though narrower), but with marginal statistical significance.

\section{2. $A P E X$}

We also observed the ${ }^{12} \mathrm{CO} J=3-2$ line of SN1987A using the Atacama Pathfinder Experiment (APEX) in Chile (European Southern Observatory proposal ID: 090.D-0515(A)). We used the SHeFI receiver (Nyström et al. 2009) at the central frequency of $345.796 \mathrm{GHz}$, and upper side band and wobbler beam switching mode with two different chopping distances of $40^{\prime \prime}$ and $45^{\prime \prime}$. The back end system has $8192 \times 2$ channels of $61 \mathrm{kHz}$ width. The two bands are $4 \mathrm{GHz}$ wide each $(1 \mathrm{GHz}$ overlapping). The system temperature was typically $200 \mathrm{~K}$. We chose $1.5 \mathrm{hr}$ (of the most stable sky conditions) of the total $4.5 \mathrm{hr}$ of data acquired over 4 days (2012 October 6, 8, 10, and 16, PWV from 0.2-0.7 mm). The focus was checked with Jupiter, $o$ Cet and R Dor; the pointing was regularly checked and updated on the nearby pointing source $\mathrm{R}$ Dor. The antenna temperature was converted to main beam temperature and then to flux in Jy using $41 \mathrm{Jy} \mathrm{K}^{-1}$ (for the $17^{\prime \prime}$ beam). We subtracted the baseline after combining all the spectra using the CLASS software and obtained an upper limit on the ${ }^{12} \mathrm{CO} J=3-2$ line of $94 \mathrm{mJy}$ $\left(5.2 \times 10^{32} \mathrm{erg} \mathrm{s}^{-1}\right)$.

\subsection{SPIRE}

The ${ }^{12} \mathrm{CO} J=4-3$ to $J=13-12$ transitions are encompassed by the Spectral and Photometric Imaging REceiver (SPIRE; Griffin et al. 2010) Fourier Transform Spectrometer (FTS; 447 to $1550 \mathrm{GHz}$ ) onboard the Herschel Space Observatory (Pilbratt et al. 2010). SN1987A was observed by SPIRE on 2012 June 12 for $\sim 4$ hr (observation ID 1342246989, proposal GT2_mbarlow_1) as part of the MESS program (Groenewegen et al. 2011) and reduced in HIPE v11. We detected two lines, $J=7-6$ and $J=6-5$, at $(7.0 \pm 2.5)$ and $(8.0 \pm 2.0) \times 10^{-18} \mathrm{~W} \mathrm{~m}^{-2}$. In luminosity, they are $(2.01 \pm 0.8)$ and $(2.4 \pm 0.6) \times 10^{33} \mathrm{erg} \mathrm{s}^{-1}$, respectively. These lines were fit with a FWHM of $2300 \mathrm{~km} \mathrm{~s}^{-1}$ (Figure 2), consistent in origin with the emission measured by ALMA. Those two CO lines fall in the lowest noise region of the entire band (the sensitivity is wavelength dependent), though we were also able to derive upper limits of $1.3-5.2 \times 10^{-17} \mathrm{~W} \mathrm{~m}^{-2}$ for the remaining lines. In increasing order of $J_{\text {up }}$ from 4 to 13 , excluding 6 and 7 , the upper limits are $1.5,1.3,1.4,2.6,3.0,4.0,3.0$, and $5.2 \times 10^{-17} \mathrm{~W} \mathrm{~m}^{-2}$.

To determine the upper limits, we took an artificial ${ }^{12} \mathrm{CO}$ input spectrum with zero continuum and all eight lines given fluxes of $8.7 \times 10^{-18} \mathrm{~W} \mathrm{~m}^{-2}$ and very narrow widths. We then broadened the lines with a Gaussian function having a FWHM of $2300 \mathrm{~km} \mathrm{~s}^{-1}$. We added the broadened ${ }^{12} \mathrm{CO}$ line spectrum, multiplied by flux scaling factors ranging from 1 to 10 , to the observed subspectra in order to judge the flux scaling factor needed for each ${ }^{12} \mathrm{CO}$ line to yield a definite detection (as judged by a line flux measurement that yielded a line flux within $30 \%$ of the input value).

\section{INTERPRETATION}

Most of the emitting ${ }^{12} \mathrm{CO}$ is expected to reside in chemically distinct clumps, composed mostly of oxygen and carbon, in the inner SN debris (McCray 1993; Jerkstrand et al. 2011). We interpret the observed ${ }^{12} \mathrm{CO}$ emission lines with a simplified model, in which a total mass $M_{\mathrm{CO}}$ of ${ }^{12} \mathrm{CO}$ molecules is found in clumps of uniform density and temperature $\left(T_{\mathrm{CO}}\right)$ that occupy a volume $V_{\mathrm{CO}}$ of the inner debris. We assume that the inner debris is confined within a freely expanding sphere of volume $V_{\max }$ defined by radius $R=v t=2000 \mathrm{~km} \mathrm{~s}^{-1} \times 25 \mathrm{yr}$. (Note that the diameter, twice this expansion radius, fully encompasses the FWHM of the line emission). The clumps occupy a fraction, $f_{\mathrm{CO}}$, of that total volume, such that $V_{\max } \times f_{\mathrm{CO}}=V_{\mathrm{CO}}$. In reality, the carbon/oxygen zone is likely composed of clumps that do vary in chemical composition, density, and temperature (Janka 2012). Because we do not resolve individual clumps, we are modeling the bulk properties that describe the sum of the ${ }^{12} \mathrm{CO}$ emission. We calculate the luminosities using the Sobolev escape probability approximation (Castor 1970), where the expressions for the line luminosity, escape probability $\left(P_{\mathrm{esc}}\right)$, and the Sobolev optical depth $\left(\tau_{S}\right)$ take simple forms in the case of freely expanding SN debris (McCray 1993, Equations (5)-(9)). Optically thin emission depends only on $M_{\mathrm{CO}}$ and $T_{\mathrm{CO}}$, whereas at high optical depths, the line luminosity is described by the optically thick limit and depends only on $V_{\mathrm{CO}}$ and $T_{\mathrm{CO}}$.

In the above model, we assume that the lines are in local thermodynamic equilibrium (LTE) with their surroundings and the populations of the rotational levels are described by an excitation temperature equal to the kinetic temperature, $T_{\mathrm{CO}}$. The higher- $J$ transitions are less likely to be in LTE; radiative decays tend to depopulate higher- $J$ rotational levels. In general, the population levels and line luminosities will depend on the product of the collision partner density (likely oxygen, carbon, $\mathrm{O}_{2}$ and other ${ }^{12} \mathrm{CO}$ molecules) and collision rate coefficients, which are unknown for these species. To make a rough estimate, we examined the collisional rate coefficients for $\mathrm{H}_{2}+\mathrm{CO}$ (Yang et al. 2010), $\mathrm{H}+\mathrm{CO}$ (Balakrishnan et al. 2002), and $\mathrm{H}_{2} \mathrm{O}+$ CO (Green 1993). The critical densities for the $J=2-1$ line are one to two orders of magnitude below the modeled density of the $\mathrm{O} / \mathrm{C}$ clumps (Jerkstrand et al. $2011 ; 1.8 \times 10^{5} \mathrm{~cm}^{-3}$ at $25 \mathrm{yr}$ ), indicating that these lines are well approximated by LTE. The rate coefficients for the proxy species vary significantly for the higher- $J$ lines, introducing considerable uncertainties in modeling the level populations. Therefore, the LTE predictions for the spectral line energy distributions (SLEDs; as shown in Figure 3) should be considered upper limits for lines above $J=3-2$; the actual line luminosities for the same physical conditions will be lower. This means that the current SPIRE upper limits cannot constrain the physical parameters of our model, but the $J=6-5$ and 7-6 detections can exclude parameters that predict low LTE luminosities.

We first consider the fluxes of the ${ }^{12} \mathrm{CO} J=1-0$ and $2-1$ lines measured by the ALMA, which are in LTE. We can determine a robust lower limit to the mass by assuming that the $J=1-0$ line is optically thin, which yields $M_{\mathrm{CO}}>0.01 M_{\odot}$ at $<10 \mathrm{~K}$. However, the line luminosity ratio $L_{2-1} / L_{1-0}=7.9 \pm 2.0$ is not consistent with the ratio $>20$ expected in the optically thin case. Instead, it is consistent with the ratio of 8 expected in the optically thick case $\left(L_{J, J-1} / L_{1,0} \approx J^{3}\right)$ in the Rayleigh-Jeans approximation, valid for temperatures above $10 \mathrm{~K}$. This case, $L_{\text {thick }}$, defines an upper limit to the line emission because the specific intensity of radiation emitted by a surface cannot exceed the Planck function, $B_{v}\left(T_{\mathrm{CO}}\right)$. The ${ }^{12} \mathrm{CO} J=3-2$ line is probably also optically thick; assuming so, we predict a luminosity of $3.4 \times L_{2-1}=3.6 \times 10^{32} \mathrm{erg} \mathrm{s}^{-1}$ and a peak flux of $65 \mathrm{mJy}$, lower than the upper limit from APEX.

As mentioned for a freely expanding volume, the resulting value of $L_{\text {thick }}$ depends only on the net volume, $V_{\mathrm{CO}}$, of the 


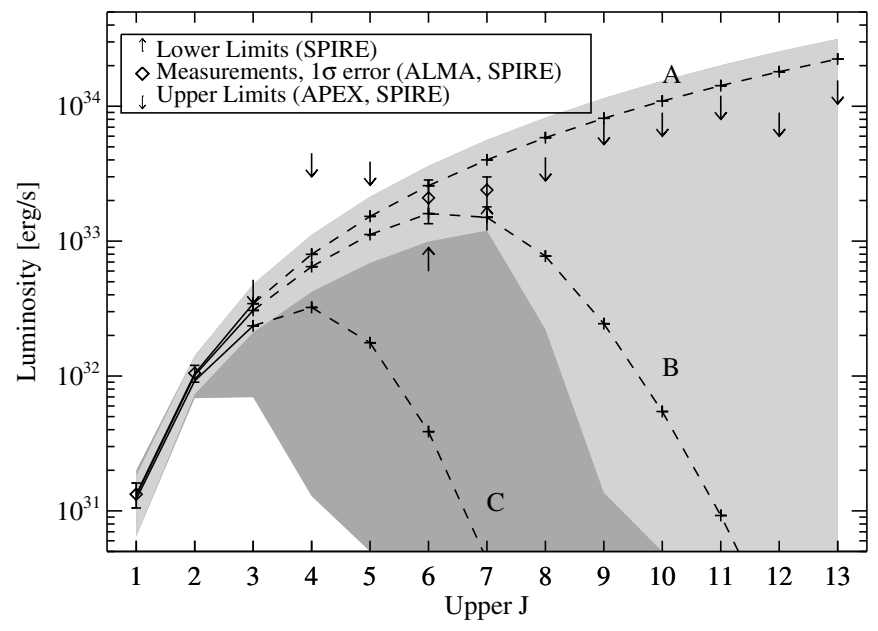

Figure 3. ${ }^{12} \mathrm{CO}$ measurements and upper limits with example spectral line energy distributions (SLEDs). Diamonds, downward arrows, and upward arrows (at the base of the arrow) indicate measurements, lower limits, and upper limits, respectively. The light gray shaded region illustrates possible LTE SLEDs consistent with ALMA measurements and SPIRE lower limits (solutions of at least $5 \%$ of the maximum likelihood solution); the dark gray region indicates additional (low- $T_{\mathrm{CO}}$, high- $f_{\mathrm{CO}}$ ) solutions that are excluded by SPIRE lower limits. Lines A, B, and C are example LTE SLEDs; lines are dashed above $J=3-2$ to indicate that LTE is an upper limit and the true luminosity is likely lower than this curve. Line A [B, C] corresponds to $T_{\mathrm{CO}}=132[27,13] \mathrm{K}$, $M_{\mathrm{CO}}=0.55[0.063,0.028] M_{\odot}, f_{\mathrm{CO}}=0.025[0.141,0.348]$. Lines A and B may both be consistent with available data despite arising from very different physical conditions; Line $\mathrm{C}$ is an example of a set of conditions that can be excluded given the SPIRE $J=6-5$ and $J=7-5$ measurements.

emitting material and the excitation temperature, independent of $M_{\mathrm{CO}}$. The product of $T_{\mathrm{CO}}$ and $V_{\mathrm{CO}}$ is $5.3 \times 10^{52} \mathrm{~cm}^{3} \mathrm{~K}$.

Figure 3 shows the ALMA and SPIRE line luminosities from this work along with example SLEDs (labeled A, B, and C), all of which are consistent with the ALMA measurements, despite arising from very different physical conditions. We cannot constrain the mass from optically thick emission, but we can find a minimum mass at which the emission is optically thick and reproduces the ALMA observations. For all combinations of temperature and $f_{\mathrm{CO}}<1$, the minimum mass is $0.01 M_{\odot}(3 \sigma$ lower limit).

Though the SPIRE lines cannot be modeled accurately, we can rule out any parameter space whose LTE luminosity does not reach the lower limits of the two measured lines. In Figure 3, the lower limits are given by the SPIRE measurements minus $2 \sigma$ (upward arrows). The parameter space excluded by the SPIRE lines is highlighted by the dark gray region, clearly excluding low- $T_{\mathrm{CO}}$ and high- $f_{\mathrm{CO}}$ solutions. The light gray region shows possible SLEDs allowed by the ALMA, APEX, and SPIRE measurements and contains only temperatures greater than $14 \mathrm{~K}$ and filling factors less than 0.35 ; the minimum mass to satisfy these conditions is still $0.01 M_{\odot}$.

\section{DISCUSSION AND CONCLUSIONS}

From the above analysis, the C/O clumps in SN1987A contain at least $0.01 \mathrm{M}_{\odot}$ of ${ }^{12} \mathrm{CO}$, an order of magnitude greater than measured in the first few years after the explosion (Liu et al. 1992). The implication is that ${ }^{12} \mathrm{CO}$ has continued to form over the past $25 \mathrm{yr}$. According to a model of the evolution of the optical and near-IR emission line spectrum (Jerkstrand et al. 2011) at $t=8 \mathrm{yr}$, the carbon/oxygen clumps have a mass of carbon plus oxygen of $0.58 M_{\odot}$ and a filling factor $f_{\mathrm{C} / \mathrm{O}}=0.02$. Such a small filling factor would imply a higher
${ }^{12} \mathrm{CO}$ temperature $(>100 \mathrm{~K})$ and at least $0.2 M_{\odot}$ of ${ }^{12} \mathrm{CO}(1 \sigma$ lower limit).

Other studies have specifically examined molecule formation within the distinct chemical zones of the ejecta. For example, a $15 M_{\odot}$ progenitor at solar metallicity may form $>0.2 M_{\odot}$ of ${ }^{12} \mathrm{CO}$, a significant amount of which is formed as early as 600 days after the explosion (Cherchneff \& Sarangi 2011). As the progenitor of SN1987A is most likely a $19 \pm 3 M_{\odot}$ blue giant with metallicity similar to the Large Magellanic Cloud (Woosley 1988, one-third to one-fourth solar), we can also compare to results from Population III stars (with zero metallicity). Cherchneff \& Dwek (2009) found similar results for a Population III $20 M_{\odot}$ progenitor, estimating $0.27 M_{\odot}$ of ${ }^{12} \mathrm{CO}$ produced on the same timescale. The velocity adopted for the $\mathrm{CO}$ model presented here matches that used in the Cherchneff \& Dwek (2009) study $\left(2242 \mathrm{~km} \mathrm{~s}^{-1}\right)$. Though our observations alone can provide only a minimum mass, the combination of the ALMA spectra results with the model computed by Jerkstrand et al. (2011) is consistent with theoretical models of molecule formation that have been untested until now.

In addition to the lower limits for temperature and mass, Herschel-SPIRE provides an upper limit to the total ${ }^{12} \mathrm{CO}$ luminosity. Recently, using the photometric capability of Herschel $(100-500 \mu \mathrm{m})$, the FIR luminosity of the debris of SN1987A was measured to be $8.45 \times 10^{35} \mathrm{erg} \mathrm{s}^{-1}$, and was attributed to thermal emission radiated by a massive reservoir of cold dust grains in the ejecta by Matsuura et al. (2011). The lack of detection with SPIRE FTS in the same wavebands provides an upper limit to the contribution from rotationally excited ${ }^{12} \mathrm{CO}$ molecules to the FIR luminosity, with ${ }^{12} \mathrm{CO}$ contributing at most $8.4 \%$. This demonstrates that emission from dust grains is still the most viable explanation for the excess FIR emission detected with Herschel.

We have presented measurements for four ${ }^{12} \mathrm{CO}$ rotational transitions in the ejecta of SN1987A, as well as upper limits for eight additional lines. The unprecedented angular resolution of ALMA firmly locates this emission as originating from the inner ejecta. The $J=2-1$ and $J=1-0$ lines are optically thick and require at least $0.01 M_{\odot}$ of ${ }^{12} \mathrm{CO}$. These results, combined with previous dust results from Herschel, illustrate a SN environment filled with cool molecules and gas merely $25 \mathrm{yr}$ after the explosion. These results are not only the first of their kind, they are also exciting as a demonstration for future work. Unlike optical observations, where the redshifted emission is largely obscured by dust, (sub)millimeter observations can see the entire velocity range of the debris. Surfaces of constant Doppler shift are planar sections of the freely expanding SN debris, and the ${ }^{12} \mathrm{CO}$ lines are sufficiently bright that we will be able to image the debris in three dimensions with resolution better than 0.'02 through Doppler tomography with the full ALMA array (Isensee et al. 2010; DeLaney et al. 2010; Larsson et al. 2013). ALMA will be able to do the same with different molecules (e.g., $\mathrm{SiO}$ ) to probe chemically distinct regions, which likely have different morphologies.

J.K. acknowledges funding from the National Science Foundation Graduate Research Fellowship Program. R.K.'s supernova research at Harvard is supported by the NSF through grant AST-1211196. J.B., P.v.H., and P.R. acknowledge support from the Belgian Science Policy office through the ESA Prodex program. M.L. acknowledges an ESO/Keele studentship. J.M. received partial support from grants AYA2009-13036C02-02 of the MICINN and PROMETEO 104/2009 of the 
Generalitat Valenciana. M. Meixner acknowledges funding support from NASA Herschel Space Center, JPL contracts \#1381522 and 1381650 and NASA NAG5-12595. The National Radio Astronomy Observatory is a facility of the National Science Foundation operated under cooperative agreement by Associated Universities, Inc. This paper makes use of the following ALMA data: ADS/JAO.ALMA\#2011.0.00273.S. ALMA is a partnership of ESO (representing its member states), NSF (USA) and NINS (Japan), together with NRC (Canada) and NSC and ASIAA (Taiwan), in cooperation with the Republic of Chile. The Joint ALMA Observatory is operated by ESO, AUI/NRAO and NAOJ.

Facilities: ALMA, Herschel, APEX

\section{REFERENCES}

Balakrishnan, N., Yan, M., \& Dalgarno, A. 2002, ApJ, 568, 443

Biermann, P. L., Chini, R., Haslam, C. G. T., et al. 1992, A\&A, 255, L5

Briggs, D. S. 1995, High Fidelity Deconvolution of Moderately Resolved Sources

Castor, J. I. 1970, MNRAS, 149, 111

Cherchneff, I., \& Dwek, E. 2009, ApJ, 703, 642

Cherchneff, I., \& Sarangi, A. 2011, in IAU Symp. 280, The Molecular Universe ed. J. Cernicharo \& R. Bachiller (Cambridge: Cambridge Univ. Press), 228

DeLaney, T., Rudnick, L., Stage, M. D., et al. 2010, ApJ, 725, 2038

Fransson, C., Larsson, J., Spyromilio, J., et al. 2013, ApJ, 768, 88

Green, S. 1993, ApJ, 412, 436
Griffin, M. J., Abergel, A., Abreu, A., et al. 2010, A\&A, 518, L3

Groenewegen, M. A. T., Waelkens, C., Barlow, M. J., et al. 2011, A\&A, 526, A162

Isensee, K., Rudnick, L., DeLaney, T., et al. 2010, ApJ, 725, 2059

Janka, H.-T. 2012, ARNPS, 62, 407

Jerkstrand, A., Fransson, C., \& Kozma, C. 2011, A\&A, 530, A45

Kjær, K., Leibundgut, B., Fransson, C., Jerkstrand, A., \& Spyromilio, J. 2010, A\&A, 517, A51

Lakićević, M., van Loon, J. T., Patat, F., Staveley-Smith, L., \& Zanardo, G. 2011, A\&A, 532, L8

Lakićević, M., Zanardo, G., van Loon, J. T., et al. 2012, A\&A, 541, L2

Larsson, J., Fransson, C., Kjaer, K., et al. 2013, ApJ, 768, 89

Liu, W., \& Dalgarno, A. 1995, ApJ, 454, 472

Liu, W., Dalgarno, A., \& Lepp, S. 1992, ApJ, 396, 679

Matsuura, M., Dwek, E., Meixner, M., et al. 2011, Sci, 333, 1258

McCray, R. 1993, ARA\&A, 31, 175

McCray, R. 2007, in AIP Conf. Proc. 937, Supernova 1987A: 20 Years After: Supernovae and Gamma-Ray Bursters, ed. S. Immler, K. Weiler, \& R. McCray (Melville, NY: AIP), 3

Ng, C.-Y., Gaensler, B. M., Staveley-Smith, L., et al. 2008, ApJ, 684, 481

Nyström, O., et al. 2009, J. Infrared Millim. Terahertz Waves, 30, 746

Panagia, N. 1999, in IAU Symp. 190, New Views of the Magellanic Clouds, ed. Y.-H. Chu, N. Suntzeff, J. Hesser, \& D. Bohlender (Cambridge: Cambridge Univ. Press), 549

Panagia, N., Gilmozzi, R., Macchetto, F., Adorf, H.-M., \& Kirshner, R. P. 1991, ApJL, 380, L23

Pilbratt, G. L., et al. 2010, A\&A, 518, L1

Rho, J., Onaka, T., Cami, J., \& Reach, W. T. 2012, ApJL, 747, L6

Woosley, S. E. 1988, ApJ, 330, 218

Yang, B., Stancil, P. C., Balakrishnan, N., \& Forrey, R. C. 2010, ApJ, 718, 1062

Zanardo, G., Staveley-Smith, L., Ng, C.-Y., et al. 2013, ApJ, 767, 98 